\title{
Association of Triglyceride Glucose-Body Mass Index and Incident Diabetes Mellitus: A Secondary Retrospective Analysis Based On a Chinese Cohort Study
}

\section{Fan Yang}

Shenzhen University First Affiliated Hospital: Shenzhen Second People's Hospital https://orcid.org/0000-0001-7686-7020

\section{Xiaohan Ding}

Shenzhen University First Affiliated Hospital: Shenzhen Second People's Hospital

\section{Zhuangsen Chen}

Shenzhen University First Affiliated Hospital: Shenzhen Second People's Hospital

\section{Yan Liao}

Shenzhen University First Affiliated Hospital: Shenzhen Second People's Hospital

\section{Miaoling Chen}

Community Medical Center

\section{Weili Yao}

Shenzhen Longhua New District People's Hospital

Qian Liang

Shenzhen People's Hospital

Haofei Hu

Shenzhen Longhua New District People's Hospital

Xinyu Wang ( $\nabla$ wxyhorse@126.com )

\section{Research}

Keywords: TyG-BMI, Incident diabetes, Nonlinearity

Posted Date: August 5th, 2021

DOl: https://doi.org/10.21203/rs.3.rs-773598/v1

License: (c) (1) This work is licensed under a Creative Commons Attribution 4.0 International License. Read Full License 


\section{Abstract}

\section{Background}

The triglyceride glucose-body mass index (TyG-BMI) has been proposed as a marker of insulin resistance (IR). However, evidence for the relationship between TyG-BMI and the incidence of diabetes mellitus remains limited. This study investigated the association between TyG-BMI and diabetes occurrence in Chinese individuals.

\section{Methods}

This retrospective study included a cohort of 204978 non-diabetic individuals using data from healthy screening program data in China between 2010 and 2016. The independent and dependent variables are TyG-BMI and incident of diabetes, respectively. Cox proportional hazards regression analysis was used to evaluate hazard ratios (HRs) and 95\% confidence intervals (95\% Cls) for the relationship between TyG$\mathrm{BMI}$ and incident diabetes. Generalized additive models were used to identify non-linear relationships. Subgroup analysis helped better understand other factors that may affect the association between TyG$\mathrm{BMI}$ and diabetes to identify potential special populations. And the data were downloaded from the DATADRYAD website.

\section{Result}

Our study indicated that the incidence of diabetes increases with the rise of TyG-BMI (HR $=1.023$, $95 \% \mathrm{CI}(1.022,1.024)$ ) after adjusting age, gender, SBP, DBP, TC, HDL, LDL, ALT, AST, Scr, smoking status, drinking status, family history. There was a nonlinear relationship between TyG-BMI and the incidence of diabetes, and the inflection point was 232.416. The effect size and confidence interval of the left and right sides of the inflection point were $1.029(1.027,1.031), 1.016(1.014,1.018), P$ for interaction < 0.0001 . Subgroup analysis showed that the correlation was stronger in the population aged $20-30$ ( $P$ for interaction $<0.0001, \mathrm{HR} 1.029,95 \% \mathrm{Cl}: 1.024$ to 1.035$)$, and the same trend was found in the following populations: age $30-40(H R=1.032)$, age $40-50(H R=1.029)$, $H D L$ (high group) ( $H R=1.024)$, $S B P<140(H R=1.025), D B P<90(H R=1.024)$, current drinker $(H R=1.031)$, and ever drinker $(H R=1.032)$.

\section{Conclusion}

This study demonstrated that increased TyG-BMI was positively correlated with incident diabetes in Chinese. TyG-BMI and incident diabetes had non-linear relationship. Before and after TyG-BMI equals 232.416 , the risk of diabetes increased by $2.9 \%$ and $1.6 \%$, respectively, when TyG-BMI increased one unit.

\section{Background}

Diabetes has become a critical worldwide healthy problem with high prevalence. The latest International Diabetes Federation (IDF) Diabetes Atlas (9th edition) indicated that about 463 million adults aged 2079 suffered from diabetes in the world in 2019[1]. It was estimated that by 2045 , the number of diabetes 
patients would reach 700.2 million[1]. China has the largest number of diabetic patients. Diabetes mellitus(DM) and its complications have resulted in a severe economic burden of mortality and disability. Thus, Early identification and prediction of diabetes are crucial.

Type 2 diabetes(T2DM) is more common among obese individuals than nonobese individuals[1]. Insulin resistance (IR) and the consequences of compensatory hyperinsulinemia are vital pathological mechanisms of diabetes mellitus and obesity[2]. Thus, recognition of IR before the manifestation of clinical diabetes mellitus is of paramount importance.

Recently, triglyceride glucose body mass index(TyG-BMI), which combines triglyceride(TG), fasting plasma glucose(FPG), and obesity status, has been considered to identify IR more reliably than TyG.[3, 4] Triglycerideglucose (TyG) index, which is estimated using the formula $\operatorname{Ln}($ fasting triglycerides $(\mathrm{mg} / \mathrm{dl}) \times$ fasting blood glucose $(\mathrm{mg} / \mathrm{dl}) / 2)$, is an alternative for identifying insulin resistance in apparently healthy subjects[5-9]. Some studies revealed the TyG index was relevant with a high risk of diabetes. TyG-BMI= TyG $\times$ BMI[3]. Body mass index (BMI) is an easily detectable, inexpensive, and non-invasive measurement parameter closely related to IR. Ectopic obesity is the biggest risk factor for type 2 diabetes[10]. A Chinese study indicates that $\mathrm{BMI}$ is also independently related to the higher risk of diabetes among young people[11]. A Korean study suggests that triglyceride glucose-body mass index is a simple and clinically useful proxy for insulin resistance in non-diabetic individuals[11]. At present, there are few articles about the relationship between TyG-BMI and diabetes.

In this study, we performed a secondary data analysis based on previously published data. In that paper, the author investigated the association of body mass index and age with incident diabetes [11]. On this secondary analysis, TyG-BMI was used as an independent variable, and outcome variables and other covariates are consistent with those in the original research. The purpose of this study was to explore the association of TyG-BMI with incident diabetes.

\section{Methods}

\section{Study population and design}

In this population-based cohort analysis of a medical program established by the Rich Healthcare Group in China between 2010 and 2016, we investigated the effect of TyG-BMI on incident diabetes. Data were downloaded from the DATADRYAD website (www.datadryad.org), allowing others to use the database for free. In keeping with the terms of service, this research cites data packets shared by Chen Ying et al.[11]. The database materials included the following variables: sex, age, BMI, drinking, smoking, family history of diabetes, low-density lipoprotein cholesterol (LDL-C), high-density lipoprotein cholesterol (HDL-C), total cholesterol (TC), TG, FPG, Serum urea nitrogen(BUN), serum creatinine (Scr), aspartate aminotransferase (AST), alanine aminotransferase (ALT), systolic blood pressure (SBP), diastolic blood pressure (DBP), incident diabetes at follow up and follow-up time. The authors declared that they had relinquished 
copyright and relevant ownership of the database in the original paper. As for ethics approval, the study was a retrospective analysis approved by the rich healthcare group review committee.

The original study enrolled 685,277 participants $\geq 20$ years old with at least two visits covering the period 2010-2016 across 32 sites and 11 cities in China (Shanghai, Beijing, Nanjing, Suzhou, Shenzhen, Changzhou, Chengdu, Guangzhou, Hefei, Wuhan, Nantong). The data we got has been filtered. Subjects with the following conditions were excluded: (1) missing information about weight, height, gender, fasting plasma glucose value at baseline; (2) extreme BMI values ( $<15 \mathrm{~kg} / \mathrm{m}^{2}$ or $\left.>55 \mathrm{~kg} / \mathrm{m}^{2}\right)$; (3) visit intervals less than 2 years; (4)participants were diagnosed with diabetes at baseline and with undefined diabetes status at follow-up. Finally, in the original study, Ying Chen et al.[11] kept 211,833 participants. We excluded missing values of baseline $T G(n=4,887)$ and zero values of baseline $T G(n=860)$ from the analysis cohort for further research. And then, TyG-BMI was calculated as the formula BMIx Ln (fasting triglycerides $(\mathrm{mg} / \mathrm{dl}) \times$ fasting blood glucose $(\mathrm{mg} / \mathrm{dl}) / 2)$. We excluded outliers of TyG-BMI(<means minus three standard deviation (SD) or $>$ means plus three $S D)(n=1,108)$. Finally, a sum of 204978 participants was selected in our study. Figure 1 depicted the participants' selection process.

\section{Data Collection And Measurements}

The original database contained participants' clinical history and lifestyle factors based on a standardized questionnaire regarding demographic characteristics, lifestyle factors, personal medical history, and family history of chronic disease. Trained staff measured height, weight, and blood pressure. Body weight was measured in light clothing with no shoes to the nearest $0.1 \mathrm{~kg}$. Height was measured to the nearest $0.1 \mathrm{~cm}$. BMI was derived from weight in kilograms divided by height in meters squared. Standard mercury sphygmomanometers measured blood pressure. Fasting venous blood samples were collected after at least a 10 hours fast at each visit. TG, TC, LDL-C, HDL-C and Plasma glucose levels were measured on an autoanalyzer (Beckman 5800). Plasma glucose levels were measured by the glucose oxidase method. The formula of the TyG index was $\mathrm{Ln}$ (fasting triglycerides $(\mathrm{mg} / \mathrm{dl}) \times$ fasting blood glucose $(\mathrm{mg} / \mathrm{dl}) / 2))$. The target-independent variable is TyG-BMI, which equals the BMI $\times$ TyG index. The dependent variable is incident diabetes, which was defined as fasting plasma glucose $\geq 7 \mathrm{mmol} / \mathrm{L}$, and/or self-reported during follow-up. As this is a retrospective cohort study, it reduced the possibility of selection bias and observation bias.

\section{Statistical analysis}

The continuous variable of the normal distribution was represented by mean with standard deviation (SD), and the continuous variable of non-normal distribution was replaced by median with interquartile range (IQR). Categorical variables were expressed as frequencies and percentages. For the handling of missing values, missing continuous variables were replaced with a mean or median depending on the distribution. Missing categorical variables could be a new categorical group. Stratified by TyG-BMI index quartiles, statistical differences of the groups were described with one-way ANOVA (normal distribution), 
Kruskal Wallis $\mathrm{H}$ (skewed distribution) test, and chi-square test (categorical variables). To explore the relationship between TyG-BMI and incident DM, univariate and multivariate Cox proportional hazard models were applied and adjusted hazard ratios (HRs) with 95\% confidence intervals (Cls). According to the recommendation of the STROBE statement, We used three models: crude model; model I adjust for: Age, Gender, SBP, DBP, Smoking Status, Drinking Status, Family History; model II adjust for: Age, Gender, SBP, DBP, Smoking Status, Drinking Status, Family History, TC, HDL, LDL, ALT, AST, Scr. Sensitivity analysis was used to ensure the robustness of data analysis. The TyG-BMI was converted into a categorical variable and calculated the $\mathrm{P}$ for trend to perform the linear trend tests. We used the Cox proportional hazards regression model with cubic spline functions to identify non-linear relationships. In addition, If there was obvious in a smoothed curve, the recursive method automatically calculates the inflection point. The associations of TyG-BMI with incident diabetes in subgroups were also studied using a stratified linear regression model and likelihood ratio test to find modifications and interactions. The subgroups were classified by age ( 20 to $<30,30$ to $<40,40$ to $<50,50$ to $<60,60$ to $<70, \geq 70$ ), gender (male vs. female), HDL( low, middle, high, not recorded), LDL( low, middle, high, not recorded), $\operatorname{SBP}(<140$, $\geq 140$ ), $\operatorname{DBP}(<90, \geq 90)$, Smoking status(current smoker, ever smoker, never smoker, not recorded), Drinking status(current drinker, ever drinker, never drinker, not recorded), Family history of diabetes(no, yes). Survival estimates and cumulative event rates were compared using the Kaplan-Meier method by using the time-to-first event for each endpoint.

All of the analyses were performed with the statistical software package R (http://www.R-project.org, The R Foundation) and Empower-Stats (http://www.empowerstats.com, X\&Y Solutions, Inc., Boston, MA). Significance was accepted at a two-tailed $\mathrm{P}<0.05$.

\section{Result}

\section{Baseline characteristics of the study participants}

Of the 211,833 subjects recruited in the former study, 204,978 participants were included in the current analysis (described in Fig. 1). The mean age of the population was $42.2 \pm 12.7$ years old, and $45.17 \%$ of participants were women. The mean year of follow-up was $3.1 \pm 0.9$ years, and 4093 participants happened diabetes during follow-up. The mean TyG-BMI was $158.7 \pm 32.4$, and the mean FPG, BMI, TG were $88.6 \pm 10.9 \mathrm{mg} / \mathrm{dl}, 23.3 \pm 3.3 \mathrm{~kg} / \mathrm{m}^{2}$ and $24.2 \pm 18.6 \mathrm{mg} / \mathrm{dl}$ respectively. Individuals in the highest TyG-BMI group (Q4) were generally older than those in the lowest TyG-BMI group (Q1) and had higher BMI, FPG, TG, SBP, DBP, TC, ALT, SCR values. What's more, with the increase of TyG-BMI value, the incidence of diabetes increased gradually. (Q1: $0.23 \%$ vs. Q2: $0.62 \%$ vs. Q3: $1.74 \%$ vs. Q4: $5.35 \%$ ). Compared with the Q1 group, the Q4 group had lower HDL levels, higher AST and LDL levels, higher rates of smoking, drinking, family history. As shown in Table 1. 
Table 1

The Baseline Characteristics of participants

\begin{tabular}{|c|c|c|c|c|c|}
\hline TyG-BMI & Q1( $\leq 167.01)$ & $\begin{array}{l}\text { Q2(167.01 to } \leq \\
191.53)\end{array}$ & $\begin{array}{l}\text { Q3(191.53 to } \leq \\
219.26)\end{array}$ & Q4(ヌ219.26) & $\begin{array}{l}\mathrm{P} \text { - } \\
\text { value }\end{array}$ \\
\hline Participants & 51245 & 51244 & 51244 & 51245 & \\
\hline Age(years) & $36.65 \pm 10.06$ & $41.26 \pm 12.13$ & $44.79 \pm 13.08$ & $46.16 \pm 13.11$ & $\begin{array}{l}< \\
0.001\end{array}$ \\
\hline $\operatorname{BMI}\left(\mathrm{kg} / \mathrm{m}^{2}\right)$ & $19.45 \pm 1.36$ & $21.98 \pm 1.20$ & $24.11 \pm 1.32$ & $27.21 \pm 2.14$ & $<.001$ \\
\hline $\mathrm{FPG}(\mathrm{mmol} / \mathrm{L})$ & $4.68 \pm 0.53$ & $4.84 \pm 0.55$ & $4.98 \pm 0.58$ & $5.17 \pm 0.63$ & $\begin{array}{l}< \\
0.001\end{array}$ \\
\hline $\mathrm{TG}(\mathrm{mmol} / \mathrm{L})$ & $\begin{array}{l}0.67(0.52- \\
0.86)\end{array}$ & $0.91(0.70-1.18)$ & $1.25(0.96-1.65)$ & $\begin{array}{l}1.90(1.40- \\
2.62)\end{array}$ & $<.001$ \\
\hline TyG-BMI & $\begin{array}{l}151.98 \pm \\
10.59\end{array}$ & $179.21 \pm 7.07$ & $204.83 \pm 7.94$ & $244.14 \pm 19.76$ & $<0.001$ \\
\hline $\mathrm{SBP}(\mathrm{mmHg})$ & $\begin{array}{l}110.87 \pm \\
13.45\end{array}$ & $116.16 \pm 14.96$ & $121.63 \pm 15.68$ & $127.36 \pm 16.40$ & $<.001$ \\
\hline $\mathrm{DBP}(\mathrm{mmHg})$ & $69.34 \pm 9.12$ & $72.00 \pm 9.74$ & $75.54 \pm 10.30$ & $79.63 \pm 11.02$ & $<.001$ \\
\hline $\mathrm{TC}(\mathrm{mmol} / \mathrm{L})$ & $4.36 \pm 0.79$ & $4.60 \pm 0.85$ & $4.82 \pm 0.88$ & $5.05 \pm 0.92$ & $<0.001$ \\
\hline ALT(U/L) & $\begin{array}{l}13.00 \\
(10.30,17.20)\end{array}$ & $\begin{array}{l}15.90(12.00- \\
21.90)\end{array}$ & $\begin{array}{l}20.00 \text { (15.00- } \\
28.60)\end{array}$ & $\begin{array}{l}28.00(19.30- \\
41.70)\end{array}$ & $<.001$ \\
\hline Scr(umol/L) & $63.93 \pm 13.20$ & $68.29 \pm 15.49$ & $72.58 \pm 14.84$ & $75.36 \pm 15.64$ & $<0.001$ \\
\hline Gender & & & & & $\begin{array}{l}<.001 \\
0.01\end{array}$ \\
\hline Male & $\begin{array}{l}14983 \\
(29.24 \%)\end{array}$ & 24120 (47.07\%) & 33452 (65.28\%) & $\begin{array}{l}39551 \\
(77.18 \%)\end{array}$ & \\
\hline Female & $\begin{array}{l}36262 \\
(70.76 \%)\end{array}$ & 27124 (52.93\%) & 17792 (34.72\%) & $\begin{array}{l}11694 \\
(22.82 \%)\end{array}$ & \\
\hline $\begin{array}{l}\text { Family } \\
\text { history }\end{array}$ & & & & & $\begin{array}{l}< \\
0.001\end{array}$ \\
\hline
\end{tabular}

Values are $n(\%)$ or mean $\pm S D$

BMI Body mass index, FPG Fasting plasma glucose, TG Triglyceride, TyG-BMI = BMI $\times \mathrm{Ln}(\mathrm{fasting}$ triglycerides $(\mathrm{mg} / \mathrm{dl}) \times$ fasting blood glucose $(\mathrm{mg} / \mathrm{dl}) / 2)$, SBP Systolic blood pressure, DBP Diastolic blood pressure, TC Total cholesterol, ALT Alanine aminotransferase, Scr Serum creatinine, HDL-C High-density lipid cholesterol, LDL-C Low-density lipid cholesterol, AST Aspartate aminotransferase. 


\begin{tabular}{|c|c|c|c|c|c|}
\hline TyG-BMI & Q1( $\leq 167.01)$ & $\begin{array}{l}\text { Q2(167.01 to } \leq \\
\text { 191.53) }\end{array}$ & $\begin{array}{l}\text { Q3(191.53 to } \leq \\
219.26)\end{array}$ & Q4(囚219.26) & $\begin{array}{l}\mathrm{P} \text { - } \\
\text { value }\end{array}$ \\
\hline NO & $\begin{array}{l}50327 \\
(98.21 \%)\end{array}$ & $50095(97.76 \%)$ & 50187 (97.94\%) & $\begin{array}{l}50179 \\
(97.92 \%)\end{array}$ & \\
\hline YES & 918 (1.79\%) & 1149 (2.24\%) & 1057 (2.06\%) & $1066(2.08 \%)$ & \\
\hline $\mathrm{HDL}(\mathrm{mmol} / \mathrm{L})$ & & & & & $\begin{array}{l}< \\
0.001\end{array}$ \\
\hline Low & $4321(8.43 \%)$ & 7255 (14.16\%) & 11440 (22.32\%) & $\begin{array}{l}15213 \\
(29.69 \%)\end{array}$ & \\
\hline Middle & $\begin{array}{l}8289 \\
(16.18 \%)\end{array}$ & 10160 (19.83\%) & 10449 (20.39\%) & 9178 (17.91\%) & \\
\hline High & $\begin{array}{l}14117 \\
(27.55 \%)\end{array}$ & 11538 (22.52\%) & 8407 (16.41\%) & $5957(11.62 \%)$ & \\
\hline Not record & $\begin{array}{l}24518 \\
(47.84 \%)\end{array}$ & $22291(43.50 \%)$ & 20948 (40.88\%) & $\begin{array}{l}20897 \\
(40.78 \%)\end{array}$ & \\
\hline LDL(mmol/L) & & & & & $<.001$ \\
\hline Low & $\begin{array}{l}13027 \\
(25.42 \%)\end{array}$ & $10258(20.02 \%)$ & 8164 (15.93\%) & 7193 (14.04\%) & \\
\hline Middle & $\begin{array}{l}8693 \\
(16.96 \%)\end{array}$ & 10309 (20.12\%) & 10299 (20.10\%) & 9935 (19.39\%) & \\
\hline High & $5080(9.91 \%)$ & 8594 (16.77\%) & $12194(23.80 \%)$ & $\begin{array}{l}13712 \\
(26.76 \%)\end{array}$ & \\
\hline Not record & $\begin{array}{l}24445 \\
(47.70 \%)\end{array}$ & 22083 (43.09\%) & 20587 (40.17\%) & $\begin{array}{l}20405 \\
(39.82 \%)\end{array}$ & \\
\hline $\operatorname{AST}(\mathrm{U} / \mathrm{L})$ & & & & & $\begin{array}{l}< \\
0.001\end{array}$ \\
\hline Low & $\begin{array}{l}10327 \\
(20.15 \%)\end{array}$ & 8482 (16.55\%) & 6057 (11.82\%) & $3722(7.26 \%)$ & \\
\hline Middle & $\begin{array}{l}7088 \\
(13.83 \%)\end{array}$ & 7635 (14.90\%) & 7815 (15.25\%) & $6243(12.18 \%)$ & \\
\hline High & 3699 (7.22\%) & $5490(10.71 \%)$ & 7956 (15.53\%) & $\begin{array}{l}11777 \\
(22.98 \%)\end{array}$ & \\
\hline
\end{tabular}

Values are $n(\%)$ or mean $\pm S D$

BMI Body mass index, FPG Fasting plasma glucose, TG Triglyceride, TyG-BMI = BMI $\times \mathrm{Ln}(\mathrm{fasting}$ triglycerides $(\mathrm{mg} / \mathrm{dl}) \times$ fasting blood glucose $(\mathrm{mg} / \mathrm{dl}) / 2)$, SBP Systolic blood pressure, DBP Diastolic blood pressure, TC Total cholesterol, ALT Alanine aminotransferase, Scr Serum creatinine, HDL-C High-density lipid cholesterol, LDL-C Low-density lipid cholesterol, AST Aspartate aminotransferase. 


\begin{tabular}{|c|c|c|c|c|c|}
\hline TyG-BMI & Q1( $\leq 167.01)$ & $\begin{array}{l}\text { Q2(167.01 to } \leq \\
191.53)\end{array}$ & $\begin{array}{l}\text { Q3(191.53 to } \leq \\
219.26)\end{array}$ & Q4(ヌ219.26) & $\begin{array}{l}\mathrm{P}- \\
\text { value }\end{array}$ \\
\hline Not record & $\begin{array}{l}30131 \\
(58.80 \%)\end{array}$ & 29637 (57.84\%) & $29416(57.40 \%)$ & $\begin{array}{l}29503 \\
(57.57 \%)\end{array}$ & \\
\hline $\begin{array}{l}\text { Smoking } \\
\text { status }\end{array}$ & & & & & $\begin{array}{l}< \\
0.001\end{array}$ \\
\hline $\begin{array}{l}\text { Current } \\
\text { smoker }\end{array}$ & $1282(2.50 \%)$ & $2161(4.22 \%)$ & $3248(6.34 \%)$ & $4841(9.45 \%)$ & \\
\hline Ever smoker & $298(0.58 \%)$ & $507(0.99 \%)$ & $768(1.50 \%)$ & $893(1.74 \%)$ & \\
\hline Never smoker & $\begin{array}{l}11551 \\
(22.54 \%)\end{array}$ & $11271(21.99 \%)$ & $11017(21.50 \%)$ & $\begin{array}{l}10307 \\
(20.11 \%)\end{array}$ & \\
\hline Not record & $\begin{array}{l}38114 \\
(74.38 \%)\end{array}$ & 37305 (72.80\%) & 36211 (70.66\%) & $\begin{array}{l}35204 \\
(68.70 \%)\end{array}$ & \\
\hline $\begin{array}{l}\text { Drinking } \\
\text { status }\end{array}$ & & & & & $\begin{array}{l}<.001 \\
0.001\end{array}$ \\
\hline $\begin{array}{l}\text { Current } \\
\text { drinker }\end{array}$ & $108(0.21 \%)$ & $209(0.41 \%)$ & $386(0.75 \%)$ & $615(1.20 \%)$ & \\
\hline Ever drinker & $1059(2.07 \%)$ & $1850(3.61 \%)$ & $2572(5.02 \%)$ & $3234(6.31 \%)$ & \\
\hline Never drinker & $\begin{array}{l}11964 \\
(23.35 \%)\end{array}$ & $11880(23.18 \%)$ & $12075(23.56 \%)$ & $\begin{array}{l}12192 \\
(23.79 \%)\end{array}$ & \\
\hline Not record & $\begin{array}{l}38114 \\
(74.38 \%)\end{array}$ & 37305 (72.80\%) & $36211(70.66 \%)$ & $\begin{array}{l}35204 \\
(68.70 \%)\end{array}$ & \\
\hline \multicolumn{6}{|c|}{ Values are $n(\%)$ or mean $\pm S D$} \\
\hline \multicolumn{6}{|c|}{$\begin{array}{l}\text { BMI Body mass index, FPG Fasting plasma glucose, TG Triglyceride, TyG-BMI = BMI } \times \text { Ln }(\text { fasting } \\
\text { triglycerides (mg/dl) } \times \text { fasting blood glucose }(\mathrm{mg} / \mathrm{dl}) / 2) \text {, SBP Systolic blood pressure, DBP Diastolic } \\
\text { blood pressure, TC Total cholesterol, ALT Alanine aminotransferase, Scr Serum creatinine, HDL-C } \\
\text { High-density lipid cholesterol, LDL-C Low-density lipid cholesterol, AST Aspartate aminotransferase. }\end{array}$} \\
\hline
\end{tabular}

\section{Univariate Analysis}

The results of the univariate analysis were shown in Table 2. Table 2 showed that men were more likely to develop diabetes than women, and age, BMI, FPG, TG, TyG-BMI, SBP, DBP, TC, LDL, ALT, AST, SCR, smoking, drinking, and family history were all positively associated with incident diabetes. 
Table 2

The results of univariate analysis

\begin{tabular}{|lll|}
\hline & Statistics & HR $(95 \% \mathrm{Cl}) \mathrm{P}$ value \\
\hline Age & $42.216 \pm 12.703$ & $1.067(1.065,1.069)<0.00001$ \\
\hline Gender & & \\
\hline Male & $112106(54.692 \%)$ & Ref \\
\hline Female & $92872(45.308 \%)$ & $0.498(0.465,0.534)<0.00001$ \\
\hline BMI & $23.186 \pm 3.241$ & $1.256(1.245,1.267)<0.00001$ \\
\hline FPG & $4.919 \pm 0.602$ & $10.572(10.109,11.057)<0.00001$ \\
\hline TG & $1.330 \pm 0.982$ & $1.281(1.268,1.294)<0.00001$ \\
\hline Family history & & \\
\hline NO & $200788(97.956 \%)$ & Ref \\
\hline YES & $4190(2.044 \%)$ & $1.741(1.487,2.037)<0.00001$ \\
\hline TyG-BMI & $195.037 \pm 36.149$ & $1.026(1.026,1.027)<0.00001$ \\
\hline SBP & $119.002 \pm 16.360$ & $1.038(1.037,1.040)<0.00001$ \\
\hline DBP & $74.128 \pm 10.788$ & $1.045(1.043,1.048)<0.00001$ \\
\hline TC & $4.707 \pm 0.897$ & $1.422(1.380,1.465)<0.00001$ \\
\hline HDL & $38520(42.697 \%)$ & $0.782(0.714,0.858)<0.00001$ \\
\hline Low & $38229(18.650 \%)$ & Ref \\
\hline Middle & $38076(18.576 \%)$ & $0.840(0.766,0.921) 0.00021$ \\
\hline High & $40019(19.524 \%)$ & $0.751(0.683,0.826)<0.00001$ \\
\hline Not record & $88654(43.250 \%)$ & $0.570(0.526,0.616)<0.00001$ \\
\hline LDL & & $1.004(1.004,1.005)<0.00001$ \\
\hline Low & $39642(18.852 \%)$ & Ref \\
\hline Middle & & $1.127(1.019,1.247) 0.02050$ \\
\hline High & & $1.659(1.510,1.822)<0.00001$ \\
\hline ALT & & \\
\hline AST & & \\
\hline
\end{tabular}




\begin{tabular}{|lll|}
\hline & Statistics & HR $(95 \%$ Cl $)$ P value \\
\hline Low & $28588(13.947 \%)$ & Ref \\
\hline Middle & $28781(14.041 \%)$ & $1.412(1.230,1.620)<0.00001$ \\
\hline High & $28922(14.110 \%)$ & $2.668(2.354,3.025)<0.00001$ \\
\hline Not record & $118687(57.902 \%)$ & $1.332(1.186,1.496)<0.00001$ \\
\hline Scr & $70.043 \pm 15.446$ & $1.006(1.005,1.007)<0.00001$ \\
\hline Smoking status & & \\
\hline Current smoker & $11532(5.626 \%)$ & Ref \\
\hline Ever smoker & $2466(1.203 \%)$ & $0.763(0.591,0.986) 0.03850$ \\
\hline Never smoker & $44146(21.537 \%)$ & $0.440(0.388,0.499)<0.00001$ \\
\hline Not record & $146834(71.634 \%)$ & $0.584(0.526,0.650)<0.00001$ \\
\hline Drinking status & & \\
\hline Current drinker & $1318(0.643 \%)$ & Ref \\
\hline Ever drinker & $8715(4.252 \%)$ & $0.462(0.335,0.638)<0.00001$ \\
\hline Never drinker & $48111(23.471 \%)$ & $0.457(0.340,0.612)<0.00001$ \\
\hline Not record & $146834(71.634 \%)$ & $0.483(0.362,0.645)<0.00001$ \\
\hline
\end{tabular}

In Fig. 2, the cumulative risk of incident diabetes Kaplan Meier curves stratified by TyG-BMI showed that the cumulative risk of diabetes increased gradually with increasing TyG-BMI. There was a significant difference in diabetes risk between the TyG-BMI quartile groups (log-rank test $P<0.0001$ ).

\section{The Multivariate Analysis Of Tyg-bmi With Dm Risk}

To evaluate group differences in the association between TyG-BMI and incident diabetes, we applied Cox proportional hazards models, and Table 3 showed the unadjusted and adjusted models. In crude model, TyG-BMI had a positive correlation with diabetes incidence (HR $=1.026,95 \%$ confidence interval (Cl):1.026 to $1.027, \mathrm{P}<0.00001$ ). We could draw the same conclusion in model I(minimally adjusted model,adjusted age, gender, SBP, DBP, smoking status, drinking status, family history) and model II(fully adjusted model,adjusted age, gender, SBP, DBP, smoking status, drinking status, family history, TC, HDL, LDL, ALT, AST, SCR). Model I (HR=1.022, 95\% Cl: 1.021 to 1.023, $P<0.00001)$, model II( $H R=1.023,95 \%$ $\mathrm{Cl}: 1.022$ to $1.024, \mathrm{P}<0.00001)$, respectively. We also performed a sensitivity analysis taking TyG-BMI as a categorical variable (quartile) at the same time and calculating $P$ for trend. The result was consistent with that of TyG-BMI as a continuous variable (trend P<0.00001). In the fully adjusted model (model II), 
the risk of diabetes in the Q4 group increased by 10.261 times compared to the Q1 group, and the trend in the quartile was significant (trend $\mathrm{P}<0.00001$ ).

Table 3

Relationship between TyG-BMI and the incident of diabetes in different models

\begin{tabular}{|llll|}
\hline Variable & Crude model $(\mathrm{HR}, 95 \% \mathrm{Cl}, \mathrm{P})$ & Model I $(\mathrm{HR}, 95 \% \mathrm{Cl}, \mathrm{P})$ & Model II $(\mathrm{HR}, 95 \% \mathrm{Cl}, \mathrm{P})$ \\
\hline TyG-BMI & $\begin{array}{l}1.026(1.026,1.027)< \\
0.00001\end{array}$ & $\begin{array}{l}1.022(1.021,1.023)< \\
0.00001\end{array}$ & $\begin{array}{l}1.023(1.022,1.024)< \\
0.00001\end{array}$ \\
\hline $\begin{array}{l}\text { TyG- } \\
\text { BMI(quartile) }\end{array}$ & & & \\
Q1 & Ref & Ref & Ref \\
Q2 & $2.967(2.405,3.660)<$ & $2.020(1.636,2.495)<$ & $2.049(1.659,2.532)<$ \\
& 0.00001 & 0.00001 & 0.00001 \\
Q3 & $8.089(6.675,9.801)<$ & $4.195(3.450,5.102)<$ & $4.437(3.644,5.403)<$ \\
& 0.00001 & 0.00001 & 0.00001 \\
\hline Q4 & $23.876(19.854,28.714)<$ & $10.562(8.730,12.779)<$ & $11.261(9.277,13.668)<$ \\
& 0.00001 & 0.00001 & 0.00001 \\
\hline P for trend & $<0.00001$ & $<0.00001$ & $<0.00001$ \\
\hline
\end{tabular}

Data in the table: HR:hazard ratio,Cl:confidence, Ref: reference, $P$ value ${ }^{*} P<0.05 * * P<0.01{ }^{\star * * P}<$ 0.001

outcome variable: diabetes

exposure variable: TyG-BMIITyG-BMI(quartile)

Crude model adjust for: None

Adjust I model adjust for: Age, Gender, SBP, DBP, Smoking status, Drinking status, Family history

Adjust II model adjust for: Age, Gender, SBP, DBP, Smoking status, Drinking status, Family history, TC, HDL, LDL, ALT, AST, Scr

Cox model Time variable: Follow up

\section{The Analyses Of The Non-linear Relationship}

Because TyG-BMI was a continuous variable, we identified the nonlinear relationship between TyG-BMI and diabetes incidence rate (adjusted age, gender, SBP, DBP, smoking status, drinking status, family history, TC, HDL, LDL, ALT, AST, SCR) by using the generalized additive model(GAM). In addition, there was an inflection point of TyG-BMI calculated by a two-piecewise linear regression model, and the inflection point was 232.416. The association between TyG-BMI and incident diabetes was positive on 
either side of the inflection point. The positive potency was slightly weaker on the right side $(\mathrm{HR}=1.016$, $95 \% \mathrm{Cl}: 1.014$ to $1.018, \mathrm{P}<0.0001)$ of the inflection point than on the left $(\mathrm{HR}=1.029,95 \% \mathrm{Cl}: 1.027$ to 1.031, $\mathrm{P}<0.0001$ ). (Table 4,Fig. 3).

Table 4

The result of two-piecewise linear regression model

\begin{tabular}{|ll|}
\hline & incident of diabetes $(\mathrm{HR}, 95 \% \mathrm{Cl}, \mathrm{P})$ \\
\hline Fitting model by standard linear regression & $1.023(1.022,1.024)<0.0001$ \\
\hline Fitting model by two-piecewise linear regression & \\
\hline Inflection point of TyG-BMI & 232.416 \\
\hline$<232.416$ & $1.029(1.027,1.031)<0.0001$ \\
$>232.416$ & $1.016(1.014,1.018)<0.0001$ \\
\hline P for log likelihood ratio test & $<0.001$ \\
\hline Cl: Confidence interval & \\
\hline $\begin{array}{l}\text { We adjusted Age, Gender, SBP, DBP, TC, HDL, LDL, ALT, AST, SCR, Smoking status, Drinking status, } \\
\text { Family history. }\end{array}$ \\
\hline
\end{tabular}

\section{The Results Of Subgroup Analyses}

Table 5 was the subgroup analysis for the correlation between TyG-BMI and diabetes incidence to explore other risks. The participants were divided into subgroups according to age, gender, HDL, LDL, SBP, DBP, smoking status, drinking status, and family history of diabetes. The association between TyG-BMI and incident diabetes was stable in family history, smoking status, and gender of patients (all $P$ values for interaction $₫ 0.05$ ). In contrast, We observed a number of interactions, including age, HDL, LDL, SBP, DBP, Drinking status (all $\mathrm{P}$ values of interaction $<0.05$ ). The relationship between TyG-BMI and diabetes was stronger in people with age $20-30(\mathrm{HR} 1.029,95 \% \mathrm{Cl}: 1.024$ to 1.035$)$, age $30-40(\mathrm{HR} 1.032,95 \% \mathrm{Cl}: 1.029$ to 1.034 ), age $40-50(\mathrm{HR} 1.029,95 \% \mathrm{Cl}: 1.027$ to 1.031$), \mathrm{HDL}$ (high group) ( $\mathrm{HR}=1.024,95 \% \mathrm{Cl}: 1.022$ to 1.026), $\mathrm{SBP}<140(\mathrm{HR}=1.025,95 \% \mathrm{Cl}: 1.024$ to 1.027$), \mathrm{DBP}<90(\mathrm{HR}=1.024,95 \% \mathrm{Cl}: 1.023$ to 1.025$)$, current drinker(HR $=1.031,95 \% \mathrm{Cl}: 1.022$ to 1.041$)$ and ever drinker(HR $=1.032,95 \% \mathrm{Cl}: 1.027$ to 1.037$)$. In addition, the relationship between TyG-BMI and diabetes risk was weaker in the people with age 60-70 (HR $1.015,95 \% \mathrm{Cl}: 1.013$ to 1.017 ), age $\geq 70$ (HR $1.013,95 \% \mathrm{Cl}: 1.011$ to 1.016 ), HDL(low group) (HR = $1.020,95 \% \mathrm{Cl}: 1.018$ to 1.021$), \mathrm{LDL}$ (middle group) ( $\mathrm{HR}=1.022,95 \% \mathrm{Cl}: 1.020$ to 1.024$), \mathrm{LDL}$ (high group) $(\mathrm{HR}=1.021,95 \% \mathrm{Cl}: 1.019$ to 1.023$), \mathrm{SBP} \geq 140(\mathrm{HR}=1.017,95 \% \mathrm{Cl}: 1.015$ to 1.019$), \mathrm{DBP} \geq 90(\mathrm{HR}=1.018$, $95 \% \mathrm{Cl}: 1.016$ to 1.021$)$ and never drinker $(\mathrm{HR}=1.022,95 \% \mathrm{Cl}: 1.020$ to 1.024$)$. 
Page 13/22 
Table 5

Effect size of TyG-BMI on diabetes in prespecified and exploratory subgroups

\begin{tabular}{|c|c|c|}
\hline Characteristic & No. of participants & Effect size(HR,95\%Cl,P) P for interacion \\
\hline Age(years) & 27301 & $<0.0001$ \\
\hline 20 to $<30$ & 80043 & $1.029(1.024,1.035)<0.0001$ \\
\hline 30 to $<40$ & 43888 & $1.032(1.029,1.034)<0.0001$ \\
\hline 40 to $<50$ & 29252 & $1.029(1.027,1.031)<0.0001$ \\
\hline 50 to $<60$ & 17278 & $1.023(1.021,1.025)<0.0001$ \\
\hline 60 to $<70$ & 7216 & $1.015(1.013,1.017)<0.0001$ \\
\hline$\geq 70$ & & $1.013(1.011,1.016)<0.0001$ \\
\hline Gender & & 0.9252 \\
\hline Male & 112106 & $1.023(1.022,1.024)<0.0001$ \\
\hline Female & 92872 & $1.023(1.021,1.024)<0.0001$ \\
\hline $\mathrm{HDL}(\mathrm{mmol} / \mathrm{L})$ & & 0.0012 \\
\hline Low & 38229 & $1.020(1.018,1.021)<0.0001$ \\
\hline Middle & 38076 & $1.023(1.021,1.026)<0.0001$ \\
\hline High & 40019 & $1.024(1.022,1.026)<0.0001$ \\
\hline Not recorded & 88654 & $1.024(1.023,1.026)<0.0001$ \\
\hline $\mathrm{LDL}(\mathrm{mmol} / \mathrm{L})$ & & 0.0222 \\
\hline Low & 38642 & $1.023(1.021,1.025)<0.0001$ \\
\hline Middle & 39236 & $1.022(1.020,1.024)<0.0001$ \\
\hline High & 39580 & $1.021(1.019,1.023)<0.0001$ \\
\hline Not recorded & 87520 & $1.024(1.023,1.026)<0.0001$ \\
\hline $\mathrm{SBP}(\mathrm{mmHg})$ & & $<0.0001$ \\
\hline$<140$ & 185128 & $1.025(1.024,1.027)<0.0001$ \\
\hline$\geq 140$ & 20958 & $1.017(1.015,1.019)<0.0001$ \\
\hline $\mathrm{DBP}(\mathrm{mmHg})$ & & $<0.0001$ \\
\hline$<90$ & 189459 & $1.024(1.023,1.025)<0.0001$ \\
\hline
\end{tabular}

Note 1:Above model adjusted for Age, Gender, SBP, DBP, TC, HDL, LDL, ALT, AST, SCR, Smoking status, Drinking status, Family history. Note 2: In each case, the model is not adjusted for the stratification variable 


\begin{tabular}{|c|c|c|}
\hline Characteristic & No. of participants & Effect size $(\mathrm{HR}, 95 \% \mathrm{Cl}, \mathrm{P}) \mathrm{P}$ for interacion \\
\hline$\geq 90$ & 16627 & $1.018(1.016,1.021)<0.0001$ \\
\hline Smoking status & & 0.1151 \\
\hline Current smoker & 11532 & $1.025(1.022,1.028)<0.0001$ \\
\hline Ever smoker & 2466 & $1.029(1.021,1.036)<0.0001$ \\
\hline Never smoker & 44146 & $1.023(1.021,1.025)<0.0001$ \\
\hline Not recorded & 146834 & $1.022(1.021,1.023)<0.0001$ \\
\hline Drinking status & & 0.0002 \\
\hline Current drinker & 1318 & $1.031(1.022,1.041)<0.0001$ \\
\hline Ever drinker & 8715 & $1.032(1.027,1.037)<0.0001$ \\
\hline Never drinker & 48111 & $1.022(1.020,1.024)<0.0001$ \\
\hline Not recorded & 146834 & $1.022(1.021,1.023)<0.0001$ \\
\hline Family history of diabetes & & 0.1543 \\
\hline No & 200788 & $1.023(1.022,1.024)<0.0001$ \\
\hline Yes & 4190 & $1.020(1.016,1.024)<0.0001$ \\
\hline \multicolumn{3}{|c|}{$\begin{array}{l}\text { Note 1:Above model adjusted for Age, Gender, SBP, DBP, TC, HDL, LDL, ALT, AST, SCR, Smoking status, } \\
\text { Drinking status, Family history. Note } 2 \text { : In each case, the model is not adjusted for the stratification } \\
\text { variable }\end{array}$} \\
\hline
\end{tabular}

\section{Discussion}

In this China's large retrospective cohort study, we found that after adjusting for many confounding factors (age, gender, SBP, DBP, TC, HDL, LDL, ALT, AST, SCR, smoking, SBP), there was a positive nonlinear correlation between TyG-BMI and risk of diabetes. The inflection point value was 232.416 , which was consistent in the direction before and after the inflection point, but the effect value was not completely consistent ([left (HR: 1.029, 95\% Cl: 1.027-1.031, P<0.0001)]; Right (HR: 1.016, 95\% Cl: 1.014-1.018, P< 0.0001)].The subgroup analysis showed that the association between TyG-BMI and diabetes risk were stronger in the following groups: age $20-30(\mathrm{HR}=1.029,95 \% \mathrm{Cl}: 1.024$ to 1.035$)$, age $30-40(\mathrm{HR}=1.032$, 95\%Cl:1.029 to 1.034 ), age $40-50$ (HR $1.029,95 \% \mathrm{Cl}: 1.027$ to 1.031$)$, high $\mathrm{HDL}(\mathrm{HR}=1.024,95 \% \mathrm{Cl}: 1.022$ to 1.026$), \mathrm{SBP}<140(\mathrm{HR}=1.025,95 \% \mathrm{Cl}: 1.024$ to 1.027$), \mathrm{DBP}<90(\mathrm{HR}=1.024,95 \% \mathrm{Cl}: 1.023$ to 1.025$)$, current drinker( $\mathrm{HR}=1.031,95 \% \mathrm{Cl}: 1.022$ to 1.041$)$, and ever drinker $(\mathrm{HR}=1.032,95 \% \mathrm{Cl}: 1.027$ to 1.037$)$.

Metabolic disorders, such as obesity, hyperglycemia, hypertension, dyslipidemia, and hyperinsulinemia, are the pathological basis of cardiovascular and cerebrovascular diseases and diabetes. In developing type 2 diabetes, decreased $\beta$ cell function and insulin resistance are the main events[12]. Adipose tissue 
is a complex and highly active metabolic and endocrine organ[13]. Elevated blood glucose and lipid levels have toxic effects on beta cells and interfere with normal glucose metabolism. Insulin resistance triggers hyperinsulinemia, and hyperinsulinemia, in turn, causes insulin resistance[2]. It is generally accepted that insulin resistance is closely related to the risk of type 2 diabetes. Clinically, the gold standard of insulin resistance is the glucose clamp test, which is inconvenient and expensive. Although homeostasis model assessment of insulin resistance (HOMA-IR) has a wide range of clinical applications, its application is limited due to its relatively high cost and low repeatability. Simental-Mendía et al.[3] Proposed the concept of the TyG index, which indicated that the TyG index could be used as an alternative index of insulin resistance in healthy subjects. Many studies have shown that the TyG index is a good alternative marker of insulin resistance.[4, 14-17] Compared with the HOMA-IR index, the TyG index has higher sensitivity in recognizing insulin resistance[15]. TyG index is regular and easy to get, including FBG and TG, which have been associated with diabetes risk $[8,9,14,18]$. In addition to the TyG index, the relationship between obesity and diabetes is also well documented. The prevalence of type 2 diabetes mellitus is rapidly increasing, in parallel with the current obesity epidemic. The incidence rate of type 2 diabetes is lower in non-obese patients[19]. BMI is a simple, economical, and useful indicator of general obesity. A cross-sectional study of the Taiwan population shows that TyG-BMI (a combination of TyG index and $\mathrm{BMI}$ ) is an effective marker for early recognition of insulin resistance[3]. A recent study involving 511 individuals indicated that TyG-BMI was a stronger predictor of IR than TyG-WC[3]. In a Nigerian cross-sectional study[20], in all 473 participants, TyG-BMI shows larger AUC for metabolic syndrome detection $(0.838,95 \% \mathrm{Cl}: 0.802-0.870)$ than TyG index $(0.796,95 \% \mathrm{Cl}: 0.757-0.831)$. After adjusting for gender, age, smoking, SBP and DBP, only the TyG index and TyG-BMI significantly predicted metabolic syndrome in men[20]. However, a Korean retrospective study involving 11,149 people showed that TyG-BMI was superior to the other parameters(TyG index, TyG-WC, TyG-WHtR) in predicting insulin resistance[21]. These conclusions are similar to ours. Firstly, consistent with previous studies, TyG-BMI was positively correlated with the incidence of diabetes. Then we further analyzed and discovered the curvilinear relationship. After adjusting for confounding factors in our study, the association between TyG-BMI and incident diabetes was nonlinear by using two piecewise linear regression models. The inflection point of GAM was 232.416 after adjusting for potential confounding factors (age, gender, SBP, DBP, TC, HDL, LDL, ALT, AST, SCR, smoking, SBP). We found that the inflection point had a stronger relationship on the left side of the inflection point. Therefore, controlling TyG-BMI is more valuable for reducing the risk of diabetes under the inflection point.

Our study has some strength: (1).To our knowledge, this is the first study to assess the association between TyG-BMI and incident diabetes in the Chinese population; (2). Compared with other researches, our sample size is relatively larger, which can better represent the Chinese people; (3). This study was a retrospective cohort study, which reduced selection bias and observation bias; (4). We found the nonlinear relationship and made a deeper discussion, and there are also more confounding factors for adjustment; (5). To make the results more robust, TyG-BMI was treated both as a continuous and categorical variable. 
The study also has some potential limitations: (1). The data was from the Rich Healthcare Group in China, representing the Chinese population, and couldn't be extended to other races and particular groups like pregnant women and children. (2). This research was based on a secondary analysis of published data, variables were limited to the original study's data, some other important variables such as hip circumference, medication history, hemoglobin A1C, physical activity, dietary factors were not included. (3). The incidence of diabetes may underestimate because of the study's diabetes definition, which did not conduct a 2-hour oral glucose tolerance test. But for such a large cohort, It is a vast project to improve participants' oral glucose tolerance test. (4). The study did not differentiate diabetes types. But these conclusions may be more applicable to type 2 diabetes which accounts for approximately 90ariablesdiabetes patients. (5). According to TyG-BMI, we only measured it at baseline, not measured over time. In the future, we can consider more variables and a longer follow-up with a more refined method.

\section{Conclusion}

The association between TyG-BMI and incident diabetes is positive and nonlinear after correcting the related confounding factors. The inflection point was 232.416 . On the left side of the inflection point, the relationship between TyG-BMI and diabetes is the most significant.

\section{Abbreviations}

$\mathrm{BMI}$

Body mass index; TyG:Triglycerideglucose index;TyG-BMI:triglyceride glucose-body mass index; IR:insulin resistance; HRs:hazard ratios; 95\%Cls:95\% confidence intervals; IDF:International Diabetes Federation; DM:Diabetes mellitus; T2DM:Type 2 diabetes; TG:Triglyceride; FPG:fasting plasma glucose; LDL-C:lowdensity lipoprotein cholesterol; HDL-C:high-density lipoprotein cholesterol; TC:total cholesterol; BUN:serum urea nitrogen; Scr:serum creatinine; AST:aspartate aminotransferase ; ALT:alanine aminotransferase; SBP:systolic blood pressure; DBP:diastolic blood pressure. GAM:Generalized additive models; SD:standard deviation. IQR:interquartile range.

\section{Declarations}

\section{Authors' contributions}

Fan Yang and Xiaohan Ding contributed to the study concept and design, researched and interpreted the data and drafted the manuscript. Zhuangsen CHEN, Yan Liao, Miaoling CHEN researched data and reviewed the manuscript. Weili YAO and Qian LIANG oversaw the progress of the project, contributed to the discussion and reviewed the manuscript. Xinyu WANG and Haofei HU are the guarantors of this work and, as such, had full access to all the data in the study and take responsibility for the integrity of the data and the accuracy of the data analysis. All authors read and approved the final the manuscript. 
Acknowledgements

Not applicable.

\section{Competing interests}

The authors declare that they have no competing interests.

\section{Availability of data and materials}

Data can be downloaded from 'DATADRYAD' database (www.Datadryad.org).

\section{Consent for publication}

Not applicable.

\section{Ethics approval and consent to participate}

In the previously published article [11], Ying Chen, et al. has clearly stated that: the study was conducted in accordance with the Declaration of Helsinki. Informed consent was obtained from all Participants.

\section{Funding}

This study was also supported in part by the International Cooperative Research Project of Shenzhen Municipal Science and Technology Innovation Council (accounts GJHZ2018041616481462).

\section{References}

1. IDF Diabetes Atlas. 9th Edition, 2019..

2. MP, C., Insulin action and resistance in obesity and type 2 diabetes. Nature medicine, 2017. 23(7): $p$. 804-814.

3. LK, E., et al., Triglyceride Glucose-Body Mass Index Is a Simple and Clinically Useful Surrogate Marker for Insulin Resistance in Nondiabetic Individuals. PloS one, 2016. 11(3): p. e0149731.

4. ZY, Z., et al., Association of triglyceride glucose index and its combination of obesity indices with prehypertension in lean individuals: A cross-sectional study of Chinese adults. Journal of clinical hypertension (Greenwich, Conn.), 2020. 22(6): p. 1025-1032.

5. LE, S., R. M and G. F, The product of fasting glucose and triglycerides as surrogate for identifying insulin resistance in apparently healthy subjects. Metabolic syndrome and related disorders, 2008. 6(4): p. 299-304.

6. SH, L., et al., Predicting the development of diabetes using the product of triglycerides and glucose: the Chungju Metabolic Disease Cohort (CMC) study. PloS one, 2014. 9(2): p. e90430.

7. JW, L., L. NK and P. HY, The product of fasting plasma glucose and triglycerides improves risk prediction of type 2 diabetes in middle-aged Koreans. BMC endocrine disorders, 2018. 18(1): p. 33. 
8. S, L., et al., The role of triglyceride glucose index in development of Type 2 diabetes mellitus. Diabetes research and clinical practice, 2018. 143: p. 43-49.

9. X, L., et al., Association between triglyceride-glucose index and risk of incident diabetes: a secondary analysis based on a Chinese cohort study : TyG index and incident diabetes. Lipids in health and disease, 2020. 19(1): p. 236.

10. T, O., et al., Ectopic fat obesity presents the greatest risk for incident type 2 diabetes: a populationbased longitudinal study. International journal of obesity (2005), 2019. 43(1): p. 139-148.

11. Y, C., et al., Association of body mass index and age with incident diabetes in Chinese adults: a population-based cohort study. BMJ open, 2018. 8(9): p. e021768.

12. EU, A., et al., Natural history of $\beta$-cell adaptation and failure in type 2 diabetes. Molecular aspects of medicine, 2015. 42: p. 19-41.

13. EE, K. and F. JS, Adipose tissue as an endocrine organ. The Journal of clinical endocrinology and metabolism, 2004. 89(6): p. 2548-56.

14. $M, Z$., et al., Cumulative increased risk of incident type 2 diabetes mellitus with increasing triglyceride glucose index in normal-weight people: The Rural Chinese Cohort Study. Cardiovascular diabetology, 2017. 16(1): p. 30.

15. F, G., et al., The product of triglycerides and glucose, a simple measure of insulin sensitivity. Comparison with the euglycemic-hyperinsulinemic clamp. The Journal of clinical endocrinology and metabolism, 2010. 95(7): p. 3347-51.

16. F, G., et al., Fasting Triglycerides and Glucose Index as a Diagnostic Test for Insulin Resistance in Young Adults. Archives of medical research, 2016. 47(5): p. 382-387.

17. NS, M.N., et al., Triglyceride glucose index as a surrogate measure of insulin sensitivity in obese adolescents with normoglycemia, prediabetes, and type 2 diabetes mellitus: comparison with the hyperinsulinemic-euglycemic clamp. Pediatric diabetes, 2016. 17(6): p. 458 - 65.

18. B, W., et al., Utility of three novel insulin resistance-related lipid indices for predicting type 2 diabetes mellitus among people with normal fasting glucose in rural China. Journal of diabetes, 2018. 10(8): p. 641-652.

19. A, A., et al., The magnitude of association between overweight and obesity and the risk of diabetes: a meta-analysis of prospective cohort studies. Diabetes research and clinical practice, 2010. 89(3): p. $309-19$.

20. TH, R., et al., Triglyceride-Glucose Index and Related Parameters Predicted Metabolic Syndrome in Nigerians. Metabolic syndrome and related disorders, 2021. 19(2): p. 76-82.

21. J, L., et al., Comparison of triglyceride glucose index, and related parameters to predict insulin resistance in Korean adults: An analysis of the 2007-2010 Korean National Health and Nutrition Examination Survey. PloS one, 2019. 14(3): p. e0212963.

\section{Figures}




\section{According to the original study:}

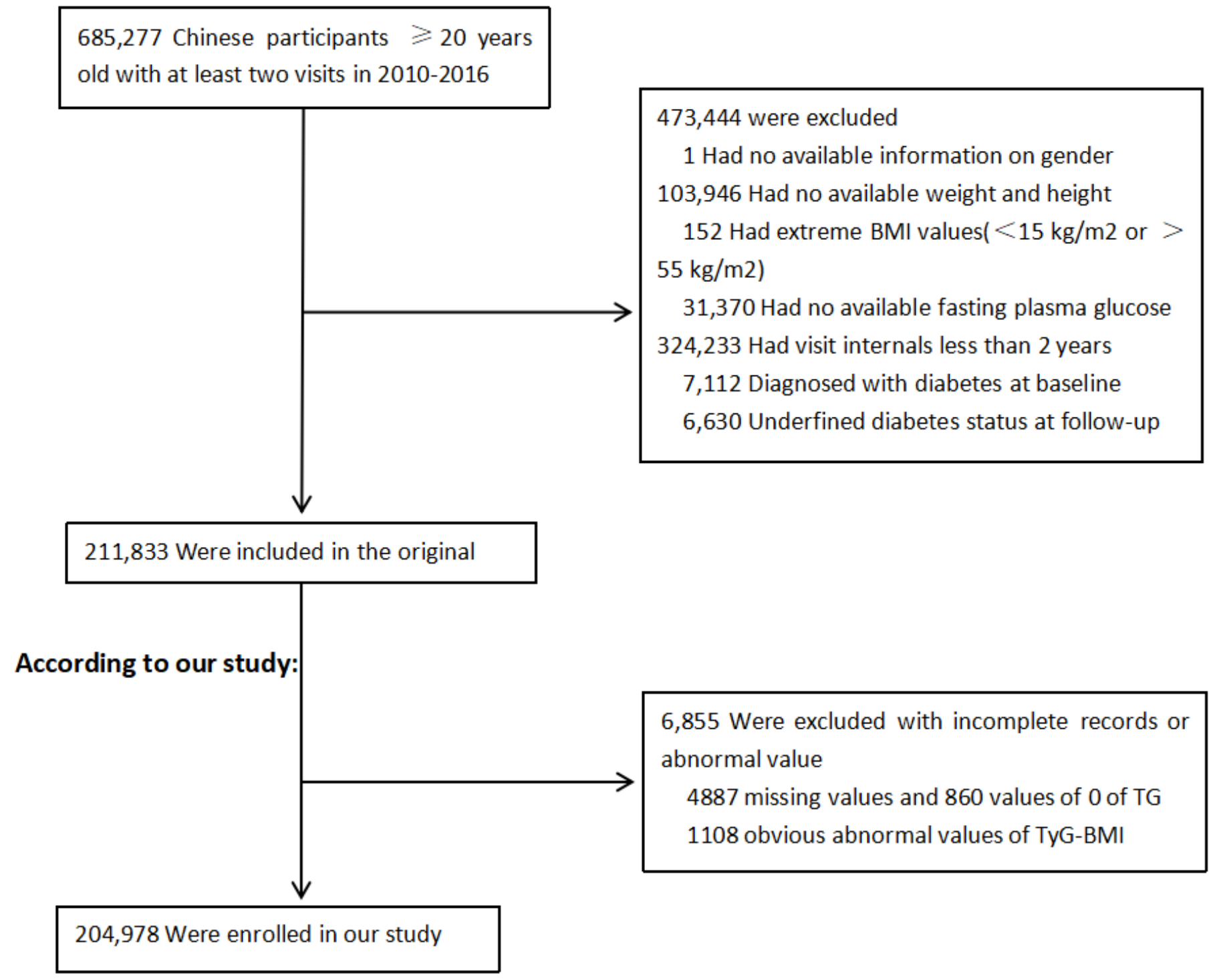

Figure 1

Flowchart of study population 


\section{DIABETES}

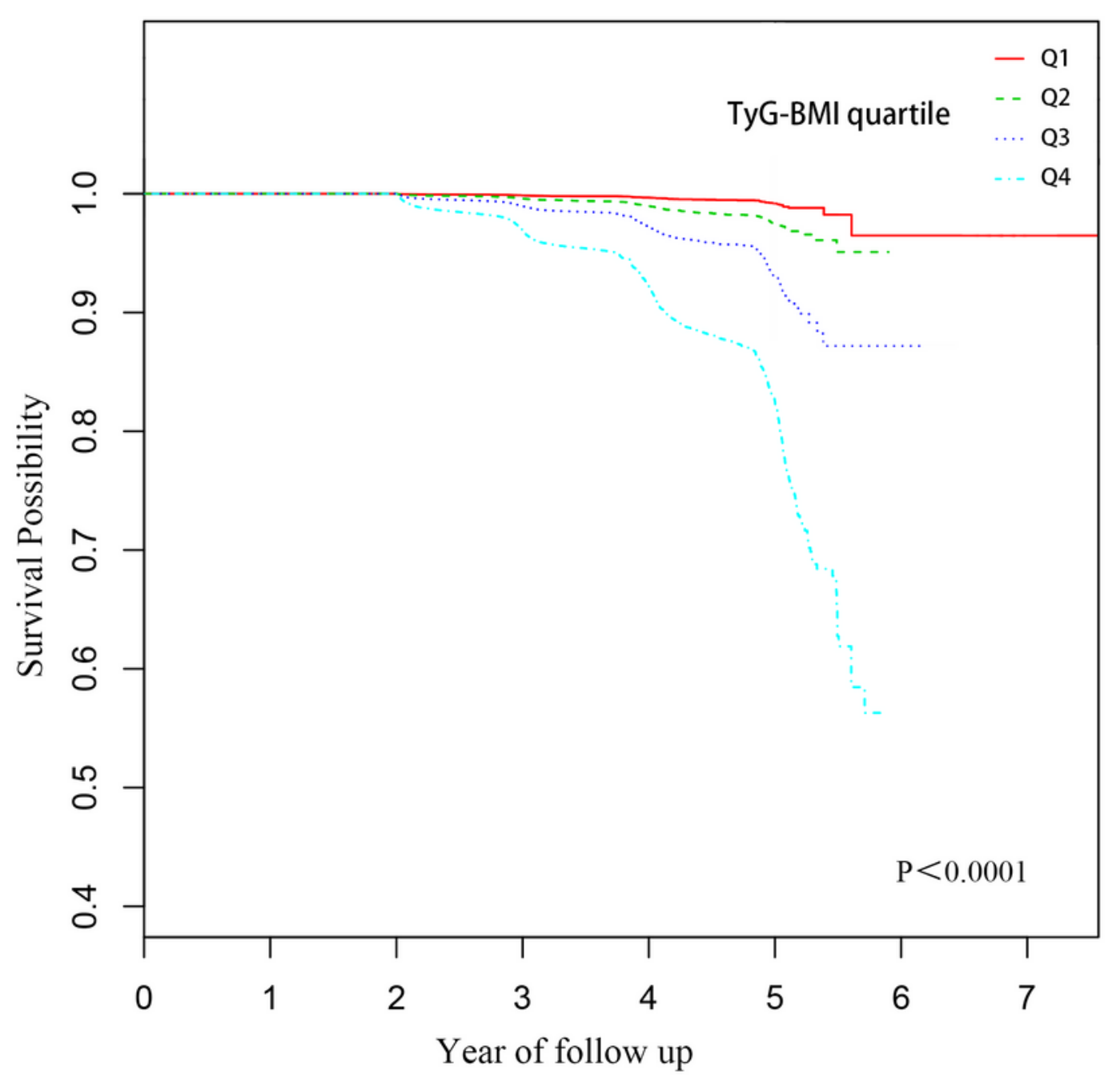

Number at risk

\begin{tabular}{|c|c|c|c|c|c|c|}
\hline - Q1 & 51245 & 26493 & 11942 & 2277 & 2 & 1 \\
\hline - Q2 & 51244 & 25383 & 11104 & 2085 & 0 & 0 \\
\hline .... Q3 & 51244 & 24724 & 10589 & 2064 & 2 & 0 \\
\hline Q4 & 51245 & 24352 & 10206 & 2198 & 0 & 0 \\
\hline & 2 & 3 & 4 & 5 & 6 & 7 \\
\hline
\end{tabular}

Figure 2

Kaplan-Meier event-free survival curve. Kaplan-Meier analysis of incident of diabetes based on TyG-BMI quartiles (logrank, $\mathrm{P}<0.0001)$. 


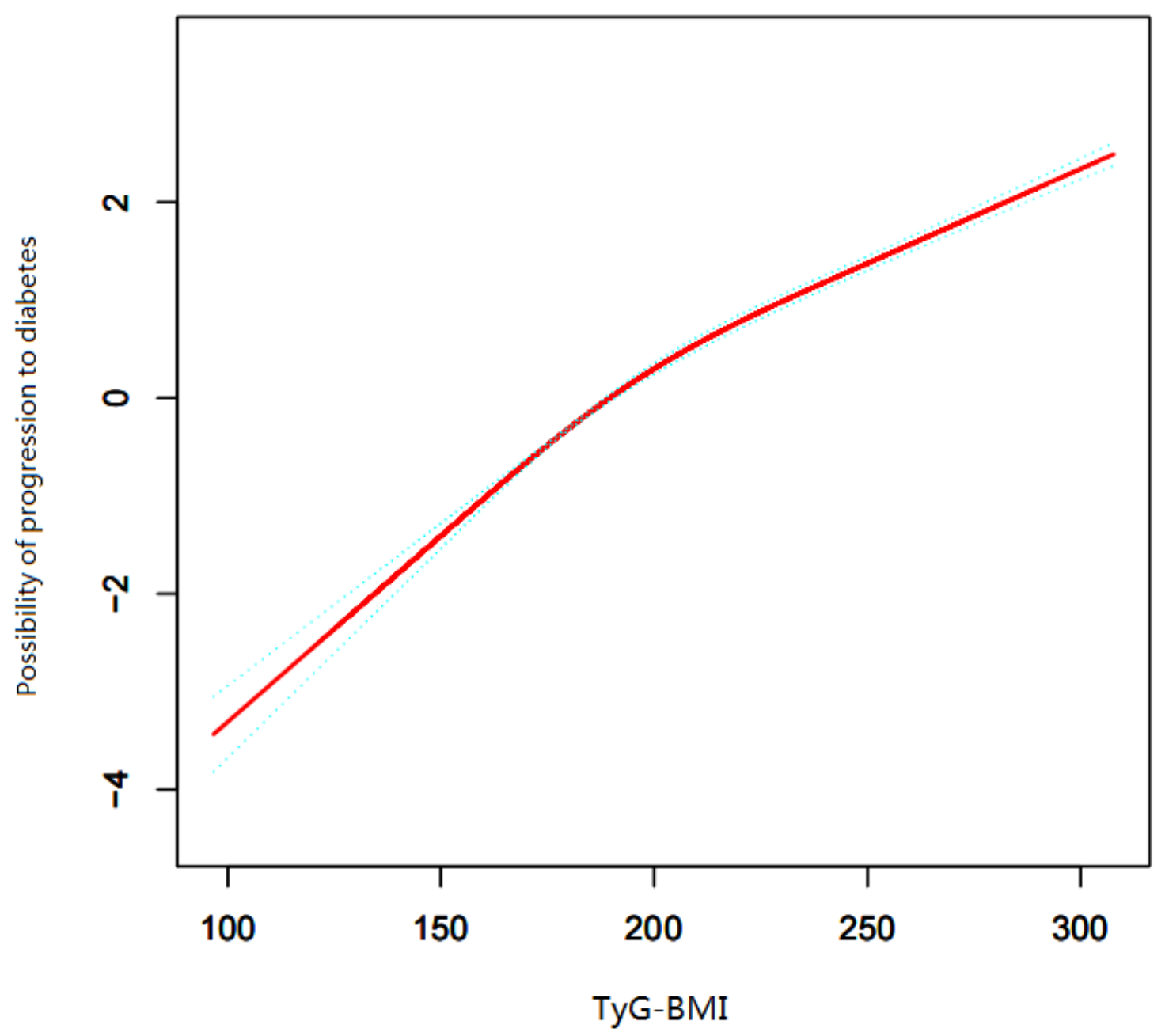

Figure 3

The non-linear relationship between TyG-BMI and incident of diabetes after adjusting for Age, Gender, SBP, DBP, Smoking Status, Drinking Status, Family History, TC, HDL, LDL, ALT, AST, SCR. 